\title{
New insights in dispersion mechanisms of carbon black in a polymer matrix under shear by rheo- optics
}

Véronique COLLIN, Innocent BOUDIMBOU and Edith PEUVREL-DISDIER *

MINES ParisTech, Centre de Mise en Forme des Matériaux (CEMEF),

UMR CNRS/Ecole des Mines de Paris N7635, BP 207, 06904 Sophia Antipolis, France

* To whom correspondence should be addressed; electronic mail: edith.disdier@mines-

paristech.fr

\begin{abstract}
The dispersion behaviour of different carbon black grades was investigated in a common elastomer matrix under shear using a transparent plate-and-plate counter-rotating shear cell coupled with an optical microscope. The objective was to investigate the effect of carbon black intrinsic characteristics (specific area and structure) on dispersion mechanisms. Shear conditions were selected in order to study independently erosion and rupture mechanisms.

The independent study of rupture and erosion mechanisms brings new findings on the respective effect of the filler intrinsic parameters on each mechanism:

- Rupture is a sudden mechanism occurring above a critical shear stress which depends on the pellet size. The rupture criterion appears not to depend on the carbon black specific area or the structure,

- Erosion proceeds via the detachment of a fixed eroded volume per strain unit and is driven by the applied shear stress and strain. Erosion is a local mechanism. The erosion rate depends on the carbon black characteristics. Faster erosion was measured for a carbon black with a higher structure at equivalent specific area or a lower specific area at equivalent structure.

This in-situ characterisation of dispersion mechanisms highlights that the effect of the carbon black characteristics on the two main dispersion mechanisms (rupture and erosion) is completely different.
\end{abstract}

Keywords: carbon black, fracture, elastomer, shear, rheo-optics 


\section{Introduction}

The degree of carbon black dispersion during mixing strongly affects the physical and mechanical properties of filled rubber parts. An effective reinforcement of the rubber matrix by the filler implies a good dispersion and distribution of the filler in the matrix. Classical reinforcing filler for rubber are carbon black and silica. They are introduced as pellets (agglomerates). Pellets are agglomerated objects with a multiscale structure. They consist of aggregates, themselves constituted by fused elementary particles. The dispersion process corresponds to the filler size reduction during the mixing operation. The minimum size which can be reached is the aggregate size. Dispersion and distribution of the filler in the polymer matrix is performed during the mixing operation in an internal mixer for example. Dispersion is possible as soon as the hydrodynamic stress induced by the flow exceeds some cohesive stress of the agglomerates. Two main mechanisms of dispersion occur during mixing, denoted as rupture and erosion. These two mechanisms are distinguished by the size of resulting fragments, the kinetics of the process and the needed energy input. Rupture is characterised by the sudden fracture of the pellet into a few fragments. Erosion is a progressive mechanism where small fragments are continuously detached from the periphery of the parent pellet. These mechanisms were first deduced from dispersion experiments performed in an internal mixer [1-2]. The use of rheo-optical techniques allows one to observe in-situ during shear the dispersion mechanisms of agglomerates [3-7]. The role of different parameters on dispersion was investigated: cohesion strength of the filler, hydrodynamic parameters, affinity between the filler and the matrix, level of infiltration of the matrix inside the agglomerate, viscosity of the matrix [4]. Most studies were conducted on model systems (agglomerates obtained by compression of fluffy powders, with a millimetre diameter range, suspended in a low viscosity Newtonian fluid). Due to the low viscosity of the matrix, the infiltration level plays a key role on the dispersion kinetics.

Dispersion processes being very sensitive to the filler/matrix system, the prediction of the dispersion step for a given system cannot be extrapolated from the knowledge of the behaviour of a model filler/matrix system or another system. In order to better understand the dispersion step in tire processing, the dispersion of commercial materials used in the tire formulation (the carbon black grade N234 and a styrene butadiene copolymer matrix) was previously investigated [6-7]. In this case, carbon black is received directly from the supplier in the shape of pellets (radius from 20 to $120 \mu \mathrm{m}$ investigated in this study). The pelletizing 
step is performed by the supplier. Pellets were thus used as received. The styrene butadiene copolymer is very viscous and highly viscoleastic. Shear conditions were defined such that erosion and rupture mechanisms could be investigated independently which is not possible in an internal mixer. The rupture mechanism characterized by the sudden breakup of the pellet into a few large fragments was observed in-situ during shear for the first time in a highly viscoelastic polymer. This study confirmed that rupture happens above a critical shear stress. However it more particularly showed that the critical stress for rupture depends on the pellet initial size (inverse proportionality). Measurements of erosion kinetics evidenced that erosion occurs beyond a critical shear stress via the continuous detachment of constant small fragment size. This means that erosion kinetics plotted as the agglomerate size reduction versus time depends on the pellet initial size (erosion is faster for a smaller pellet size). However erosion kinetics plotted as the eroded volume versus time do superimpose whatever the pellet initial size is. The mechanism is governed by the shear stress and the strain subjected to the pellet. Dispersion criteria and kinetics were used to define a mathematical model which was then used to model dispersion in an internal mixer [8]. Results were obtained on a single filler/matrix couple and stressed the role of hydrodynamic parameters on dispersion mechanisms of carbon black in this polymer matrix. However these results did not consider the role of the carbon black intrinsic properties (specific area and/or structure) on dispersion mechanisms. Dispersion studies performed in internal mixers with typical materials for the tire industry showed that the filler intrinsic characteristics affect dispersion kinetics: a carbon black with a larger structure or a lower specific area appears to be easier to disperse [9-12]. Using rheo-optics and a model system (agglomerates obtained by compression of fluffy powder, with a millimeter diameter range, suspended in a low viscosity Newtonian fluid), Li et al. (1995) [13] found a different result. In their case, they compared the behaviour of agglomerates with different structures but at equivalent densities and same elementary particle size. They found that high structure carbon black agglomerates were found more difficult to erode than agglomerates of low structure carbon black at equivalent tensile strength. The different result is explained by the authors by the fact that they worked at fixed density, whereas a pelletized high structure carbon black for rubber industry has a lower density than a pelletized low structure one which could explain the easier dispersibility of the pelletized high structure black in the case of carbon black supplied for rubber industry. We would like to address this point in the present paper via direct and independent observations of both dispersion mechanisms. 
This paper is devoted to the determination of the role of the filler intrinsic parameters such as the specific area and the structure on dispersion mechanisms in the case of commercial products used in the tire industry. This was done by comparing the dispersion behaviour of different grades of carbon black characterized by different specific areas and structures. A rheo-optical approach was used in this work. Shear conditions were chosen to study independently rupture and erosion mechanisms and thus to determine the role of the filler intrinsic parameters on each mechanism. We will show that rupture and erosion mechanisms are affected differently by the filler intrinsic characteristics.

\section{Experimental section}

\subsection{Materials}

The polymer used is this study is a styrene-co-butadiene rubber (SBR, 25E produced by Michelin), grade used in tire industry. Two SBR matrices were used. They corresponded to the same grade but came from two different batches and referred to SBR1 and SBR2 in the following. The rheological behaviour of the SBR matrices was measured by oscillatory experiments using a RMS800 Rheometrics rheometer (25mm-diameter plates). Measurements were performed in the linear regime either at 110 or $140^{\circ} \mathrm{C}$ depending on the SBR batch. The rheological behaviour of both matrices is shown in Figure 1.

\section{Figure 1}

The two batches were characterized by a different rheological behaviour. The first batch SBR1 was more viscous and more elastic than SBR2. This explains that dispersion measurements were performed at $140^{\circ} \mathrm{C}$ in SBR1 and at $110^{\circ} \mathrm{C}$ in SBR2. In these thermal conditions, the two batches had similar rheological behaviours.

A polybutadiene rubber matrix (BR, Neocis-BR40 from Enichem) was also used for some erosion measurements in order to probe the effect of the infiltration of the matrix into the filler on the erosion kinetics. This matrix was chosen for its very close rheological behaviour at $140^{\circ} \mathrm{C}$ with SBR1 but its faster infiltration ability into silica relatively to SBR (see Figure 6 in the following).

The dispersion behaviour of different carbon black grades was investigated. The filler characteristics are summarized in Table 1. 


\section{Table 1}

These carbon blacks differ in their relative specific area and structure. The specific area is determined by the adsorption of specific molecules (for example cetyltrimethyl ammonium bromide, CTAB). This measurement is related to the reverse of the size of elementary particles constituting the aggregate. The structure is representative of the way the elementary particles are arranged inside the aggregate. It is classically estimated by the measurement of the volume of oil or dibutyl phthalate (DBP) absorbed by $100 \mathrm{~g}$ of filler. A low structure carbon black is characterized by a compact arrangement whereas a high structure carbon black by a branched arrangement. The concept of structure and specific area relatively to the aggregate arrangement is illustrated in Figure 2. Five of the carbon grades were selected for their similar specific area but different structure and vice versa in order to investigate independently the effect of the specific area or the structure on the dispersion mechanisms. N326 and N347 have similar specific areas but different structures and were used to determine any effect of structure on dispersion mechanisms. On the other side N234, N327 and N160 have similar structures and different specific areas. The last two carbon black grades (N134 and N772) were chosen for their different intrinsic parameters and used in the last part of this paper to probe the relationship proposed between the erosion efficiency and the intrinsic properties of the carbon black.

All carbon grades were received in the shape of pellets of various sizes. The pelletizing step is part of the carbon black production process. In the present study, carbon black pellets were used as received from the supplier and not submitted to any particular mechanical nor thermal treatment before their use.

One silica grade (Micropearl Zeosil Z1115MP from Rhodia) was also used to probe the infiltration ability of SBR and BR matrices into the pellet. This silica grade was chosen for its similar specific area (CTAB) as compared to carbon black N234 (see Table 1).

\section{Figure 2}

\subsection{Experimental set-up}

Experiments were conducted on the transparent counter-rotating shear cell and following the procedure described in our previous work $[6-7,15]$.

The transparent counter-rotating shear cell coupled with an optical microscope was used to observe the carbon black dispersion mechanisms while the pellet was suspended in the 
polymer matrix and submitted to a shear flow. The apparatus consists of two transparent parallel glass plates rotating in opposite directions (Figure 3). The advantage of this geometry is that the relative velocities of the two plates can be adjusted so that the velocity of the suspended particle can be set to zero in the reference framework of the laboratory. This allows us to observe using an optical microscope the behaviour of the same particle suspended in a matrix under shear for a long period of time. The apparatus is equipped with ovens in order to be able to work from room temperature up to $180^{\circ} \mathrm{C}$. Observations are performed in the plane containing the vorticity axis and the flow direction at 110 or $140^{\circ} \mathrm{C}$ depending on the SBR matrix used.

A stainless steel ring was fixed on the lower plate in order to confine the polymer flow inside the parallel plate geometry. This ring aims at extending the shear rate range where observations under shear can be performed. The ring avoids the matrix ejection and pushes the development of elastic instabilities which disturb the flow to higher shear rates [15]. Shear rates up to $150 \mathrm{~s}^{-1}$ can be investigated.

\section{Figure 3}

\subsection{Procedures}

\subsubsection{Dispersion tests}

Samples consisted of a sandwich of two thin films of SBR with a few pellets of carbon black randomly set in between.

Attention was paid to obtaining reproducible results and thus to all factors which may affect the hydrodynamic stress or the cohesion stress. This includes not only the mechanical settings of the shear cell (glass plate parallelism, colinearity of the axes of rotation, concentricity of the outer ring with both plates, zero gap and rotation speed of the plate calibrations) which are necessary to define a correct shear rate, but also the following parameters:

- the pellet diameter relatively to the gap dimension: the vicinity of the shear cell walls may affect the normal stress submitted to the agglomerate and consequently its dispersion rate. This was evidenced experimentally by Ponchaitaward et al. via the determination of different dispersion rates for two sizes of agglomerate subjected to the same macroscopic shear stress [16]. The numerical simulation of the flow around a solid particle allowed us to define the condition under which there is no wall effect on the particle. If the $\mathrm{R} / \mathrm{h}<0.2$ (where $\mathrm{R}$ is the radius of the pellet and $\mathrm{h}$ is the distance between the center of the particle and the closest glass plate), the pellet is only 
subjected to the applied macroscopic shear stress and not to additional stresses due to the vicinity of the shear cell walls [17],

- the pellet shape: only pellets with a shape as spherical as possible were characterized,

- the contact time between the filler and the matrix before the dispersion test: the pellet being porous, the matrix may infiltrate into the agglomerate and modify its cohesion and the way the stress is transmitted through the agglomerate and induce changes in the kinetics of dispersion $[5,18]$. Most experiments were conducted with the same contact time between the filler and the matrix prior to any shear application: after insertion of the sample in the shear cell and setting the gap, the sample was left at rest for 45 minutes in order to have a homogeneous temperature. A given shear history was then submitted to the sample, conditions in which the dispersion mechanisms was characterized. The potential effect of the matrix infiltration inside the pellet is discussed in the following for each dispersion mechanism,

- the pre-shear history submitted to the pellet before the dispersion characterisation: In order to have reproducible results, critical conditions for rupture, for example, were always determined on "fresh samples", which are pellets directly observed after the sample preparation and not submitted to any previous shear histories.

As described in our previous paper [6], different shear histories were applied in order to study rupture or erosion mechanisms independently:

- Rupture was obtained by gradually increasing the shear rate until the rupture mechanism was observed. The shear rate at which the rupture occurs is considered as the critical shear rate $\left(\dot{\gamma}_{\mathrm{C}}^{\mathrm{R}}\right)$. The critical shear stress for rupture ( $\left.\tau_{\mathrm{C}}^{\text {Rupture }}\right)$ corresponds to the shear rate multiplied by the viscosity of the SBR at this shear rate for the given temperature $\left(\eta\left(\dot{\gamma}_{\mathrm{C}}^{\mathrm{R}}, \mathrm{T}\right)\right)$ :

$\tau_{\mathrm{C}}^{\text {Rupture }}=\dot{\gamma}_{\mathrm{C}}^{\mathrm{R}} \cdot \eta\left(\dot{\gamma}_{\mathrm{C}}^{\mathrm{R}}, \mathrm{T}\right)$

- Erosion kinetics was measured by following the gradual and continuous size reduction of the carbon black agglomerate as a function of time for a given shear rate. Following the data on the rupture mechanism, the shear rate of investigation was chosen in a range where due to the pellet size, rupture was not possible for the applied shear rate. According to our previous results found on erosion, erosion data were represented as the eroded volume versus time or strain unit $(\gamma=\dot{\gamma} . t)$ :

$\mathrm{R}(\mathrm{t})^{3}-\mathrm{R}_{0}{ }^{3}=\mathrm{f}(\gamma)$ 
where $R_{0}$ is the intitial pellet size and $R$ is the pellet time at time t.

\subsubsection{Infiltration tests}

Infiltration kinetics of the SBR1 and BR matrices were measured into silica agglomerates at $140^{\circ} \mathrm{C}$. Porous silica agglomerates present the particularity to appear as black objects when observed by transmission optical microscopy if they contain air and become transparent as soon as a liquid penetrates inside. The silica Zeosil Z1115MP was chosen for its similar specific area as N234 carbon black grade. Measurements were performed in the counterrotating shear cell but at rest. The same protocol as for dispersion experiments was thus used for the sample preparation. Practical details for the infiltration kinetics determination can be found in reference 19.

\section{Results}

\subsection{Rupture mechanisms}

\subsubsection{Rupture criterion}

An example of the rupture mechanism of a N234 carbon pellet under shear is shown on Figure 4.

\section{Figure 4}

The rupture condition is experimentally determined as the critical shear rate at which the rupture mechanism is observed. This measurement is performed for different pellet sizes in order to know the rupture condition variation with the pellet size. As dispersion is assumed to occur if the hydrodynamic shear stress is larger than the cohesion strength of the pellet, the rupture criterion is expressed as the critical shear stress (using eq. 1). We found in our previous study that the critical shear stress for rupture was inversely proportional to the pellet size in the case of N234 carbon black in a SBR matrix [6]. However, as it is depicted in Figure 5, variations of the critical shear rate for rupture with the pellet size and of the critical shear stress respectively show similar tendencies. The rupture condition determined on a single filler type in a single matrix does not allow us to discriminate on whether the rupture criterion should be based on the applied shear rate or the applied shear stress.

\section{Figure 5}

For this purpose, measurements were performed in this work on the same carbon black (N234) in two different SBR matrices. Since the two SBR matrices only differ in their 
rheological behaviour (same chemical composition and thus affinity with carbon black), similar criteria for rupture should be found for the two matrices. Results are reported in Figure 5 where critical conditions for rupture are reported as the critical shear rate versus the initial pellet size (Fig. 5.a) and secondly as the critical shear stress (Fig. 5.b).

These measurements show that if the critical shear rates for rupture for the same pellet size are different in the two matrices, rupture data do superimpose when they are represented as the applied shear stress. These measurements prove that the applied shear stress is one of the hydrodynamic key parameters for the rupture mechanism. Dispersion data are in the following expressed in terms of shear stress and do not indicate in which SBR matrix the experiments were performed.

\subsubsection{Potential infiltration of SBR}

The pellets being porous, they can be infiltrated by the matrix. This is true even if the matrix is very viscous (case of SBR in this study) [19]. The level of infiltration depends on the contact time between the filler and the matrix [20]. This infiltration and more specifically the infiltration level modifies the cohesion strength of the agglomerate and the way the stress is transmitted through the agglomerate. This induces changes in the kinetics of dispersion [5, 18]. However, in the present case, the matrix being very viscous, the infiltration time can be very long and needs to be compared to the time for the dispersion experiment.

Figure 6 shows the infiltration kinetics of the SBR1 matrix into silica at $140^{\circ} \mathrm{C}$. This figure shows that in the case of SBR1, no infiltrated layer could be detected by optical microscopy before 1000 minutes of contact time between the matrix and the filler. The infiltration of a 50 $\mu \mathrm{m}$ radius micropearl was not total after 3000 minutes. Such a long infiltration in two steps was already reported by Astruc et al. for elastomer matrices [19]. The affinity of SBR towards carbon black is higher than for silica, which means that the infiltration into carbon black pellet must be quicker. However due to the huge time necessary for the SBR infiltration into silica and regarding the large size of the pellets considered during rupture experiments, we assumed that rupture measurements were performed on dry (not infiltrated) pellets. This was confirmed by the fact that variations of the contact time (around the 45 minutes defined in the protocol) did not change the critical stress for rupture for a given pellet size.

\section{Figure 6}




\subsubsection{Effect of carbon black characteristics on rupture}

The dispersion behavior of N160, N326 and N347 carbon black grades was investigated using the same protocol as for the N234 grade. The rupture mechanism observed for the different grades showed similar features as the ones observed for the N234 grade:

- rupture occurs beyond a critical shear stress,

- the critical stress depends on the initial pellet size. The larger the initial pellet size is and the easier it is to disperse the agglomerate by rupture.

The exact dependence of the critical stress for rupture with the initial pellet size is reported in Figure 7 for the different carbon black grades.

\section{Figure 7}

This figure shows that the critical stresses for rupture for the different carbon black grades measured for similar pellet sizes are the same within experimental errors. The specific area or structure of the carbon black (at least for the investigated carbon blacks) does not seem to affect the critical condition for rupture. The criterion for rupture is found to depend on the pellet size but not on the carbon intrinsic characteristics. The log-log plot of the rupture criterion shows that the critical macroscopic shear stress is proportional to $\mathrm{R}_{0}^{-\mathrm{X}}$ :

$$
\tau_{C}^{\text {Rupture }} \propto R_{0}^{-X}
$$

where $\mathrm{X}$ varies between 0.75 and 0.8 .

We previously reported an inverse proportionality for the N234 grade in SBR [6] however complementary measurements performed in this study allows a better determination of the power law exponent.

Interpretation of the non dependence of the rupture criterion on the carbon black characteristics:

The non-dependence of the critical stress for rupture is not an intuitive phenomenon. This condition can be estimated either by considering the balance between the hydrodynamic stress and the cohesion stress or by considering an energetic balance. The two approaches are considered successively in the following.

The critical shear stress for rupture can be seen as the stress necessary to separate the agglomerate into two fragments (not necessarily of equal dimensions). Horwatt et al. [21] related the cohesion strength of a fragment relatively to the agglomerate parent to the number 
of bonds between the fragment to the agglomerate parent $\left(\mathrm{N}_{\mathrm{b}}\right)$, the mean interparticle force $(\mathrm{H})$ and the size of the fragment to be detached $(\mathrm{R})$ :

$$
\sigma_{\text {Cohesion }}=\frac{\mathrm{N}_{\mathrm{b}} \mathrm{H}}{\pi \mathrm{R}^{2}}
$$

The mean interparticle force can be considered as constant for the different carbon black grades of this study. The exponent of the power law dependence of the critical stress for rupture with the agglomerate size is only predicted by this model if we assume that severed fragments have a size close to the agglomerate size $\left(\mathrm{R}=\mathrm{R}_{0}\right)$ and the number of connecting bonds to be fractured is proportional to the agglomerate initial size:

$$
N_{b} \propto R_{0}^{2-X}
$$

This corresponds to an intermediary case of agglomerate between a dense one where the number of bonds to be severed is proportional to the surface area of fracture $N_{b} \propto R_{0}^{2}$ [21] and a sparse one where the number of bonds is small and does not depend on the agglomerate size [22]. If this relationship is valid, the cohesion strength does not depend anymore on parameters characterizing the aggregate level but only on the agglomerate size. This would explain that the critical shear stress for rupture does not depend on the carbon black characteristics.

The second approach considers the energy balance for the crack development. According to Griffith's fracture criterion, the fracture grows if the strain energy supplied by the hydrodynamic stress is greater than the energy required for the crack growth. Based on this energy balance and considering the fractal dimension of the aggregate, Zaccone et al. [23] developed a fracture model for dense aggregates with high fractal dimension (case of the present carbon black grades [24]). They established a power law dependence between the critical stress for rupture and the agglomerate size where the exponent depends on the fractal dimension but not on the size of the elementary particles thus not on the specific area. The fact that, the fractal dimension is very similar for carbon blacks of different grades [24] could explain that the rupture criterion does not depend on the carbon black grade.

The two interpretations of the rupture criterion do not have the same physical meanings. The approach based on a stress balance considers the number of bonds to be severed and this model should apply if we assume that the fragment size is close to the agglomerate size and the number of bonds is nearly proportional to the agglomerate size. The energetic approach is 
based on the energy required for a crack to propagate. This approach is quite attractive since it follows the classical approach of rupture mechanics with the initiation of a crack and the crack propagation. The result of this work is that the condition for the crack propagation is the same for the investigated carbon black grades. This does not mean that the way the pellet breaks (crack propagation paths) is similar. The crack propagation paths must correspond to the weakest points (defects in the pellet) [25]. The way the three carbon grades fractured under shear (number and size of fragments) looked similar however no systematic measurement of the fragment size distribution was performed.

\subsection{Erosion mechanism:}

\subsubsection{Erosion kinetics}

An example of the pellet size reduction due to the erosion process under shear is shown in Figure 8.

\section{Figure 8}

Our previous work on erosion of N234 grade in the SBR matrix showed that erosion is possible if the shear stress is larger than a critical value, proceeding via the detachment of a constant volume per strain unit, and that the eroded volume depends on the applied shear stress and the quantity of strain units [6]. This means that the pellet size reduction is slow for large pellets and becomes faster when the pellet size decreases (Figure 8.b). The erosion process was found to be well described by a single erosion law:

$$
\mathrm{R}_{0}^{3}-\mathrm{R}_{\mathrm{t}}^{3}=\alpha\left(\tau-\tau_{\mathrm{C}}^{\text {Erosion }}\right) \dot{\gamma} \mathrm{t}
$$

where $\mathrm{R}_{0}^{3}-\mathrm{R}_{\mathrm{t}}^{3}$ is the eroded volume with $\mathrm{R}_{0}$ the initial pellet size and $\mathrm{R}_{\mathrm{t}}$ its size at time $\mathrm{t}, \alpha$

is the erosion efficiency, $\tau$ is the applied shear stress, $\tau_{c}^{\text {Erosion }}$ is the critical shear stress for erosion, $\dot{\gamma}$ is the applied shear rate and $\mathrm{t}$ is the shearing time.

This law takes into account the initial agglomerate size, the applied shear stress and the quantity of strain. It involves two parameters characteristic of the filler/matrix system: the erosion efficiency and the critical shear stress for initiating erosion.

\subsubsection{Effect of infiltration}

As for rupture, erosion in the SBR matrix was assumed to occur on dry (not infiltrated) pellets. In order to probe the effect of an infiltrated layer on the erosion process, erosion kinetics was measured in the BR matrix. This matrix was chosen for its much faster 
infiltration into silica (see Figure 6). Four different contact times were probed between 15 and 60 minutes. The time zero corresponded to the moment when the temperature control indicated the set temperature. Once the contact time was reached, a constant shear rate was applied and the resulted erosion process was measured. Figure 9 compares erosion kinetics of the N234 carbon black grade (same pellet size, $\mathrm{R}_{0}=34 \mu \mathrm{m}$ ) at the same shear rate $\left(5 \mathrm{~s}^{-1}\right)$.

\section{Figure 9}

These measurements show that the erosion rate (defined as the slope of the eroded volume per strain unit) is faster as the contact time and thus the infiltrated layer is increased. The faster erosion for a thin infiltrated layer is explained by Yamada et al. [15] by the detachment of the infiltrated layer. As the contact time is increased, the infiltrated layer should be larger which is in agreement with a higher erosion rate.

The fact that the erosion rate varies with the contact time (infiltration depth) in the BR matrix confirms that infiltration does not play any role in the case of the SBR matrix. If infiltration would play a role, for the same contact time, the infiltration depth should be larger for smaller pellet sizes [20]. In this case, for the same shear rate, the erosion rate should vary with the pellet size (via the difference in infiltrated depth) at least during the first step of erosion. This is not what is observed in the case of the SBR matrix. As shown in our previous paper, erosion data for different initial pellet sizes at a constant shear rate superimpose when plotted as the eroded volume versus time (Figure 8 in reference 6). This means that the erosion rate at fixed shear rate is constant whatever the pellet initial size and that measurements were performed on dry pellets.

\subsubsection{Effect of carbon black characteristics}

The dispersion behaviour of the different grades of carbon black was compared using the protocol designed for erosion experiments. The continuous detachment of small fragments from the outer surface of the parent agglomerate was observed for all the carbon grades under the application of a constant shear rate. Erosion kinetics was more specifically measured for the N234, N326 and N347 carbon grades. A comparison of erosion kinetics measured on the three types of carbon black at the same shear stress of $120 \mathrm{kPa}$ (corresponding to a $10 \mathrm{~s}^{-1}$ shear rate) is depicted in Figure 10. This shear stress value was selected so that the only possible dispersion mechanism is erosion for the considered pellet sizes. Measurements were performed on pellets with similar initial sizes. Following eq. 6, kinetics is plotted as the eroded volume versus the applied strain (Figure 10.a). 


\section{Figure 10}

This figure shows that the erosion mechanism although induced by the same shear stress proceeds at different erosion rates for the three carbon black grades. The comparison of the behaviour of N234 and N347 grades (different specific areas but similar structures) indicates faster erosion for the N347 grade characterized by a lower specific area. The comparison of the behavior of N347 and N326 grades (different structures but similar specific areas) depicts faster erosion for the carbon black N347 with a larger structure. This shows that the erosion process depends on the carbon black intrinsic properties.

In order to determine the parameters of the erosion law (critical shear stress for erosion and the erosion efficiency in equation 6), erosion kinetics were measured at different shear stresses for each carbon black grade. The variation of the erosion rate with the applied shear stress for the three carbon black grades is shown in Figure 10.b.

The erosion process for the grades N326 and N347 shows similar features as for the N234 grade :

- erosion proceeds via the detachment of a constant volume of matter per strain unit,

- this erosion rate $\mathrm{k}$ varies linearly with the applied shear stress and depends on the carbon black grade,

- there is a critical shear stress for initiating erosion in all cases.

The slope of the erosion rate versus stress curve corresponds to the erosion efficiency $(\alpha)$ and the intercept of the curve with the X-axis to the critical shear stress for erosion ( $\tau_{\mathrm{C}}^{\text {Erosion }}$ ) (Eq. 6). Erosion efficiencies and critical stresses for erosion values are reported in Table 2.

\section{Table 2}

The critical stresses for erosion for the three carbon black grades are in the same order of magnitude. The order of magnitude of the critical stress for erosion is only important to know whether erosion is possible or not and to be compared with the critical shear stress for rupture. The comparison with critical shear stress for rupture values shows that erosion and rupture can happen simultaneously for pellets larger than $70 \mu \mathrm{m}$ in radius. For this order of magnitude of pellet size, although both mechanisms do happen, the size reduction due to erosion is negligible relatively to the one due to rupture.

The main information for the dispersion process is the erosion efficiency and its variation with the carbon black characteristics. The effect of the carbon black grade on the erosion 
efficiency is more obvious. The two to two comparison of the erosion efficiencies for different grades shows that the erosion kinetics is larger for carbon black grades with the lower specific area (carbon black N347, when grades of similar structures N347 and N326 are compared) and the larger structure (carbon black N347, when grades of similar specific areas N234 and N347 are compared). A quicker dispersion for a carbon black with a lower specific area or a larger structure is a classical result for industrial carbon black grades dispersed in an internal mixer [9-11]. Relatively to these studies, this work allows us to differentiate the effect of the filler characteristics on each dispersion mechanism. The present measurements demonstrate that the carbon characteristics do not affect these mechanisms in the same way: no effect on the condition for rupture but an effect on erosion.

Explanation of the dependence of the erosion efficiency on the carbon black characteristics:

The present result is different from the results of $\mathrm{Li}$ et al. based on rheo-optical dispersion experiments on compressed carbon black agglomerates suspended in a low viscosity matrix [13]. However $\mathrm{Li}$ et al. compared two carbon black agglomerates characterized by a different structure but with equivalent density and same specific area (same elementary particles). Donnet et al. [24] proposed to use the porous volume determined by the DBP adsorption to calculate the apparent density of carbon black. Since the porous volume (determined per 100g of silica) via the DBP adsorption includes inter-aggregate voids and voids between agglomerates, the IS2 index based on mercury porosimetry (considering voids with a radius lower than $4 \mu \mathrm{m}$ ) is preferred to estimate the porous volume inside the pellet and calculate the apparent density of the pellets:

$$
\rho_{\text {app }}=\left(\frac{\text { IS2 }}{100}+\frac{1}{\rho_{\mathrm{CB}}}\right)^{-1}
$$

where IS2 in $\mathrm{cm}^{3} / 100 \mathrm{~g}, \rho_{\mathrm{CB}}$ is the specific density of carbon black, $\rho_{\mathrm{CB}}=1.95 \mathrm{~g} / \mathrm{cm}^{3}$.

The apparent pellet densities of the three carbon black grades used in this study were calculated using the IS2 index. Values are reported in table 1. Based on this calculation, the two carbon black grades with similar structure (N234 and N347grades) indeed have similar density. Their difference in terms of erosion efficiency is well explained by their different specific area and thus the size of their elementary particles constituting the aggregates. The erosion efficiency parameter corresponds to the effect of the applied stress on the erosion rate that is the quantity of eroded volume which is removed per strain unit (for a fixed shear rate). If we assume that the fragment to be detached is the aggregate, the size of the elementary 
particles and their arrangement inside the aggregate (that is the structure) determines the volume to be detached. The volume detached during one pellet revolution is greater for a lower specific area carbon and explains the faster erosion. At equivalent specific area (N326 and N347 grades), the two carbon blacks have a different structure and a different density which explains their different behaviour in erosion. For a fixed size of elementary particles, a higher structure corresponds to a more complex arrangement and thus a less dense aggregate occupying a larger volume which also leads to faster erosion. The result found by Li et al. concerns a different case where agglomerates with similar density, same specific area but different structures are compared. According to the authors high structure agglomerates formed by compression may result in more interlocked structures than by pelletization which explains the more difficult erosion in their case of a high structure carbon black.

For all carbon black agglomerates (pelletized or compressed agglomerates), the erosion rate depends on the applied shear stress and the quantity of strain units.

\subsubsection{Relationship between the erosion kinetics and the carbon black intrinsic parameters}

Different attempts were already made to define empirical laws relating parameters characterizing the dispersion rate to the carbon black characteristics [10-11]. Based on our measurements and existing laws, we propose an empirical law expressing the variation of the erosion efficiency $\alpha$ versus the structure (defined by the DBP value) and the specific area (defined by the CTAB value). Our data were found to obey the following empirical relationship:

$$
\alpha=\mathrm{A} \sqrt{\mathrm{CTAB}}+\mathrm{B} \frac{\mathrm{DBP}}{\sqrt{\mathrm{CTAB}}}
$$

where A and B are proportionality constants determined for the erosion efficiency parameter $\alpha$ expressed in $\mu \mathrm{m}^{3} / \mathrm{kPa}$.strain unit, CTAB in $\mathrm{m}^{2} / \mathrm{g}$ and DBP in $\mathrm{ml} / 100 \mathrm{~g}$. In this case, $\mathrm{A}=2.7$ $10^{-7}$ and $\mathrm{B}=1.310^{-6}$. The dimension of the constants was not indicated for sake of simplicity.

Based on this equation, the variation of the erosion efficiency with the structure (DBP) is represented in Figure 11 for different values of specific area (noted S in this figure).

\section{Figure 11}

The validity of this relationship was checked for two other carbon black grades N772 and N134. Estimating the erosion efficiency of these two carbon blacks is interesting since the specific area and the structure play an opposite effect on erosion. The N772 grade is assumed 
to be easy to disperse due its low specific area but difficult if one considers its low structure. This is the contrary for the N134 with its high specific area and its high structure. Erosion efficiencies were measured for these two carbon blacks. Table 3 reports the erosion efficiency parameters measured experimentally and estimated using Eq. 8. There is a good agreement between the erosion efficiency calculated by the empirical law and the experimental values. It also shows that although the N134 and N772 carbon black grades are very different, they present similar erosion efficiencies.

\section{Table 3}

\subsection{Application to a mixing operation}

Although measured on isolated pellets, the determined criteria and laws for dispersion bring some information on what happens in an internal mixer as far as dispersion is concerned:

- The dependence of the critical shear stress for rupture with the cluster size is consistent with what happens during a mixing operation. If a constant critical shear stress for rupture is assumed (as predicted by the planar rupture model [26-27]), this means that, for a given shear stress larger than the condition, all pellets (whatever their size) would be ruptured and would break down to the level of the smallest entity that is the aggregate size. Dispersion should thus be very rapid. This is of course not consistent with what is observed in practice [2,12]. This is due to the higher resistance to rupture of the small pellets. This size dependence of the critical condition for rupture thus seems to be in line with the industrial experience.

- It is obvious that rupture is more efficient to decrease the pellet size than erosion. However, the rupture mechanism is only active at the beginning of the mixing process down to a critical agglomerate size (defined by the applied shear stress). Below this size erosion is the only possible dispersion mechanism to reduce the agglomerate size.

- Although not discussed in this way in this paper, the erosion process is governed by the energy supplied to the mix. The shear stress multiplied by the strain corresponds to an energy per volume unit. The final dispersion state thus depends on the mixing energy which includes the effect of the mixing time.

- The present study highlights that the condition for the first dispersion step (dominated by the rupture mechanism) is not affected by the carbon black characteristics whereas the second step dominated by the erosion mechanism is. 


\section{Conclusions}

The effect of the carbon black intrinsic characteristics on dispersion mechanisms and criteria was investigated by direct observation of isolated clusters suspended in a SBR matrix under shear using a transparent counter-rotating shear cell coupled with an optical microscope. It was the first time that the effect of intrinsic parameters could be probed independently on rupture and erosion mechanisms. The observation and determination of dispersion criteria and laws allow us to show that the variation of the carbon black intrinsic properties does not affect in the same way the two dispersion mechanisms:

- The critical shear stress for initiating rupture mechanism varies inversely with the pellet size. This condition for rupture was found not to be affected by the carbon black intrinsic parameters.

- On the contrary, erosion which is a more local mechanism is affected by the specific surface and the structure of the carbon black pellets. Its kinetics does depend on the carbon black intrinsic properties. Erosion is faster for a low specific area carbon black at equivalent structure and a high structure carbon black at equivalent specific area. This dependence is in agreement with results obtained from internal mixer studies, where dispersion results from both mechanisms at the same time [9-11].

This type of data brings some insights on:

- the physics of the dispersion mechanisms which is linked to the internal structure of the pellet,

- and the dispersion process during a mixing operation.

This work provides a set of pertinent and workable dispersion criterion and laws that could be incorporated in a complete modelling of the flow in an internal mixer in the case of industrial products [8].

\section{Acknowledgments}

This work gathers results from different $\mathrm{PhD}$ studies. The $\mathrm{PhD}$ of Véronique Collin was part of a larger project supported by the European Community in the framework of the "Competitive and Sustainable Growth" program. The PhD of Innocent Boudimbou is granted and supported via the International Competitiveness Cluster Lyon \& Rhône-Alpes Chemicals/Environnement (AXELERA). The authors wish to thank partners of both projects 
amoung which MFP Michelin, Rhodia, Snecma Propulsion Solide, ThyssenKrupp Elastomertechnik, Optigrade-TechPro, Polyflow, Cesame (U.C. Louvain), LPMG of Mines de Saint Etienne, LRMP from Saint-Etienne university and LTDS of E.N.S. Lyon and T.U. Lodz for their fruitful collaboration. Michelin is acknowledged for supplying the products and the characterization of the fillers.

The authors wish to thank B. Cantaloube and P. Navard for stimulating discussions during this work.

\section{References}

1. Bolen, W. R.; Colwell, R. E. Soc Plast Eng J 1958, 14, 24.

2. Shiga, S.; Furuta, M. Rubber Chem Technol 1985, 58, 1.

3. Rwei, S. P.; Manas-Zloczower, I. Feke; D. L., Polym Eng Sci 1990, 30, 701.

4. Rwei, S. P.; Manas-Zloczower, I.; Feke, D. L. Polym Eng Sci 1991; 31, 558.

5. Feke, D.L. In Handbook of Powder Technology in Volume 11 Granulation; Salman, A.D., Hounslow, M.J., Sevelle, J.P.K., Ed.; Elsevier : New York, 2007, Chap. 18.

6. Collin, V.; Peuvrel-Disdier E. Elastomery 2005, 9, 9.

7. Collin, V.; Peuvrel-Disdier E. In European Rubber Research - Practical Improvements of the Mixing Process, Paderborn, Germany, 25-26 janvier 2005, 219.

8. Collin, V.; Peuvrel-Disdier E.; Alsteens, B.; Legat, V.; Avalosse, T.; Otto, S. Metwally, H.M. In ANTEC 2006 Proceedings of the 64th Annual Conference, Charlotte, NorthCarolina, USA, 2006, 908.

9. Dizon, E.S. Rubber Chem Technol 1976, 49, 12.

10. Cotten, G. R. Rubber Chem Technol 1984, 57, 118.

11. Coran, A. Y.; Donnet, J.-B. Rubber Chem Technol 1992, 65, 998.

12. Leblanc, J. In Rhéologie des élastomères et leur mise en forme. ARTEL : Namur, 1996, 295.

13. Li, Q.; Feke, D.L.; Manas-Zloczower, I. Rubber Chem Technol 1995, 68, 836.

14. Melson, J.A. In Basic rubber testing : selecting mecthods for a rubber test program. J.S. Dick. ASTM international, Wet Consholhocken, 2003.

15. Boudimbou, I.; Roux, C.; Collin, V.; Peiti, C.; Navard, P.; Peuvrel-Disdier, E. Rhéologie 2010, 18, 33.

16. Ponchaitaward, C.; Manas-Zloczower, I.; Feke, D.L. Chem Eng Sci 2003, 58, 1859. 
17. Bikard, J.; Ménard, P.; Budtova, T.; Peuvrel-Disdier, E. Comput Mat Sci 2006, 37, 517.

18. Yamada, H.; Manas-Zloczower, I.; Feke, D. L. Powder Technol 1997, 92, 163.

19. Astruc, M.; Collin, V.; Rusch, S.; Navard, P.; Peuvrel-Disdier, E. J Appl Polym Sci 2004, 91, 3292

20. Bohin, F.; Manas-Zloczower, I.; Feke, D. L. Rubber Chem Technol 1994, 67, 602.

21. Horwatt, S. W.; Manas-Zloczower, I.; Feke, D. L. Powder Technol 1992, 72, 113.

22. Bohin, F.; Manas-Zloczower, I.; Feke, D.L. Chem Eng Sci 51, 1996, 5193.

23. Zaccone, A.; Soos, M.; Lattuada, M.; Bäbler, M.U.; Morbidelli, M. Phys Rev E, 2009, 79, 061401

24. Donnet, J.B.; Bansal, R.C; Wang, M.J. In Carbon black: Science and technology, Marcel Dekker, Inc.: New York, 1993, Chap. 3.

25. Kendall, K. Powder Technol 1988, 31, 28.

26. Bagster, D. F.; Tomi, D. Chem. Engng Sci. 1974, 29, 1773.

27. Rumpf, H. In Agglomeration, W. A. Knepper ed.: New York, 1962, 379. 


\section{CAPTIONS FOR THE TABLES:}

Table 1: Characteristics of the carbon black and silica grades used in this study. Data were determined by Michelin.

Table 2: $\quad$ Erosion parameters of the carbon black grades N234, N326 and N347 in SBR. $\alpha$ is the erosion efficiency and $\tau_{\mathrm{C}}^{\text {Erosion }}$ is the critical stress for erosion.

Table 3: Erosion efficiencies of the carbon black grades N134 and N772 in SBR: comparison of the measured and calculated (Eq. 6) parameters. 


\section{CAPTIONS FOR THE FIGURES:}

Figure 1: Complex viscosity $\mid \eta *$ (a.) and storage modulus G’ (b.) against pulsation curves for the two SBR matrices. Empty symbols represent the rheological behaviour of SBR1 measured at $140^{\circ} \mathrm{C}$ and solid symbols the behaviour of SBR2 measured at $110^{\circ} \mathrm{C}$.

Figure 2: Schematic representation of the concept of the specific area and the structure on the arrangement of elementary particles inside the aggregate. Representation adapted from [14].

Figure 3: Counter-rotating shear cell. The diameter of the upper glass plate is $40 \mathrm{~mm}$.

Figure 4: Example of rupture sequence of a N234 carbon black pellet $\left(\mathrm{R}_{0}=50 \mu \mathrm{m}\right)$ in SBR1 at $140^{\circ} \mathrm{C}$ under shear $\left(\dot{\gamma}=50 \mathrm{~s}^{-1}\right)$.

Figure 5: Rupture criteria of the N234 carbon black grade in both SBR matrices represented as:

a. the critical shear rate versus the pellet size,

b. the critical shear stress versus the pellet size.

Figure 6: $\quad$ a. Example of infiltration of SBR1 into silica, $\mathrm{R}_{0}=44 \mu \mathrm{m}$ at $140^{\circ} \mathrm{C}$.

b. Comparison of infiltration kinetics of SBR1 and BR in Z1115MP micropearls $\left(\mathrm{R}_{0}=44 \mu \mathrm{m}\right.$ in SBR1, $\mathrm{R}_{0}=38 \mu \mathrm{m}$ in $\left.\mathrm{BR}\right)$ at $140^{\circ} \mathrm{C}$. Infiltration kinetics are plotted as the radius of the non-infiltrated part normalized by the micropearl radius versus time.

Figure 7: Log-log representation of the critical shear stress for rupture versus the pellet size of the N234, N326, N347 carbon black grades in SBR.

Figure 8: Sequence of erosion of a N234 carbon black cluster in SBR1: $\mathrm{R}_{0}=22 \mu \mathrm{m}$, $\dot{\gamma}=6 \mathrm{~s}^{-1}, 140^{\circ} \mathrm{C}:$

a. Sequence of images

b. Size reduction with time 
Figure 9: Erosion kinetics measured in the BR matrix at $140^{\circ} \mathrm{C}$ for different contact times between the pellets of carbon black N234 for the same pellet size, $\mathrm{R}_{0}=$ $34 \mu \mathrm{m}$, and the same shear rate $5 \mathrm{~s}^{-1}$

Figure 10: Erosion kinetics data of the N234, N326, N347 carbon black grades in the SBR matrix for an applied shear stress of $117 \mathrm{kPa}$. Kinetics plotted as:

a. the eroded volume versus strain,

b. the erosion rate versus the applied shear stress.

Figure 11: Variation of the erosion efficiency with the carbon black structure (DBP) and specific area (S) in SBR following Eq. 7. 


\begin{tabular}{|c|c|c|c|c|c|}
\hline $\begin{array}{c}\text { Carbon } \\
\text { black } \\
\text { grade }\end{array}$ & Producer & $\begin{array}{c}\text { Specific } \\
\text { area } \\
\text { CTAB } \\
\left(\mathrm{m}^{2} / \mathrm{g}\right)\end{array}$ & $\begin{array}{c}\text { Structure } \\
\text { DBP } \\
(\mathrm{ml} / 100 \mathrm{~g})\end{array}$ & $\begin{array}{c}\text { Porosity } \\
\text { IS2 } \\
\left(\mathrm{cm}^{3} / 100 \mathrm{~g}\right)\end{array}$ & $\begin{array}{c}\text { Apparent } \\
\text { density } \\
\rho_{\text {app }} \\
\left(\mathrm{g} / \mathrm{cm}^{3}\right)\end{array}$ \\
\hline N234 & Cabott & 119 & 125 & 123 & 0.57 \\
\hline N326 & Cabott & 82 & 70 & 71 & 0.82 \\
\hline N347 & Cabott & 90 & 122 & 110 & 0.62 \\
\hline N160 & Evonik & 153 & 130 & & \\
\hline N134 & Cabott & 132 & 126 & & \\
\hline N772 & Cabott & 29 & 70 & & \\
\hline Z1115MP & Rhodia & 114 & & & \\
\hline
\end{tabular}

Collin et al., Table 1 


\begin{tabular}{|c|c|c|}
\hline $\begin{array}{c}\text { Carbon black } \\
\text { grade }\end{array}$ & $\alpha$ & $\begin{array}{c}\tau_{\mathrm{C}}^{\text {Erosion }} \\
\left(\mu \mathrm{m}^{2} / \mathrm{g}\right)\end{array}$ \\
\hline $\mathrm{kPa})$ \\
\hline $\mathrm{N} 234$ & $1.8 .10^{-5}$ & 71 \\
\hline $\mathrm{N} 326$ & $1.3 .10^{-5}$ & 72 \\
\hline $\mathrm{N} 347$ & $2.0 .10^{-5}$ & 65 \\
\hline
\end{tabular}

Collin et al., Table 2 


\begin{tabular}{|c|c|c|}
\hline $\begin{array}{c}\text { Carbon black } \\
\text { grade }\end{array}$ & $\begin{array}{c}\alpha \text { measured } \\
\left(\mu \mathrm{m}^{2} / \mathrm{g}\right)\end{array}$ & $\begin{array}{c}\alpha \text { calculated } \\
\left(\mu \mathrm{m}^{2} / \mathrm{g}\right)\end{array}$ \\
\hline $\mathrm{N} 134$ & $1.71 .10^{-5}$ & $1.72 .10^{-5}$ \\
\hline $\mathrm{N} 772$ & $1.78 .10^{-5}$ & $1.82 .10^{-5}$ \\
\hline
\end{tabular}

Collin et al., Table 3 


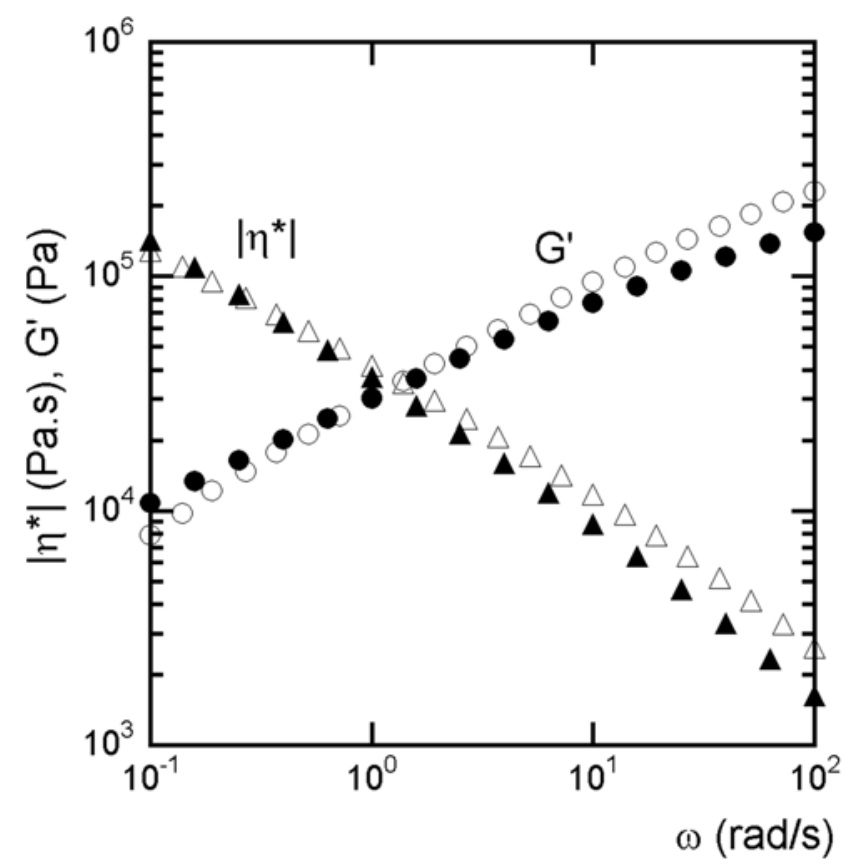

Figure 1

Collin et al., Journal of Applied Polymer Science 


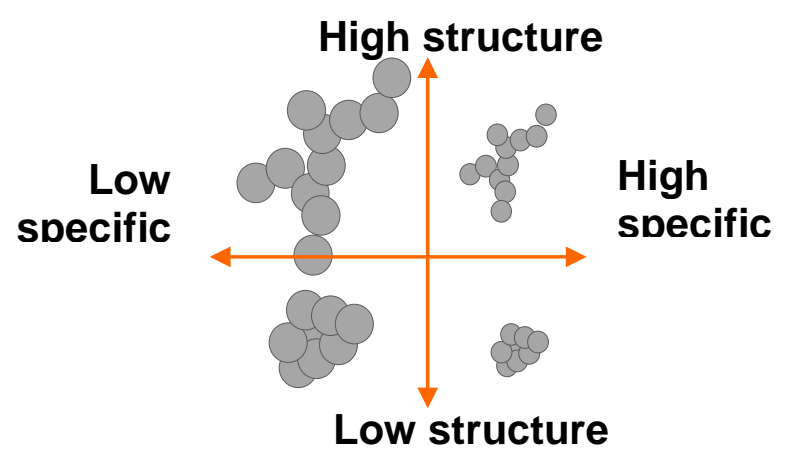

Figure 2

Collin et al., Journal of Applied Polymer Science 


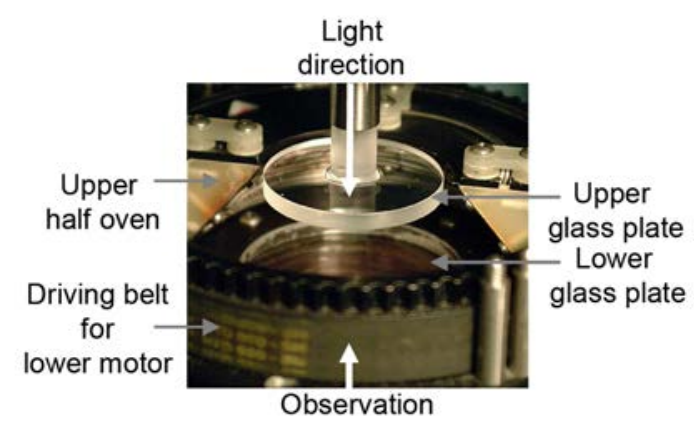

\section{Figure 3}

Collin et al., Journal of Applied Polymer Science 


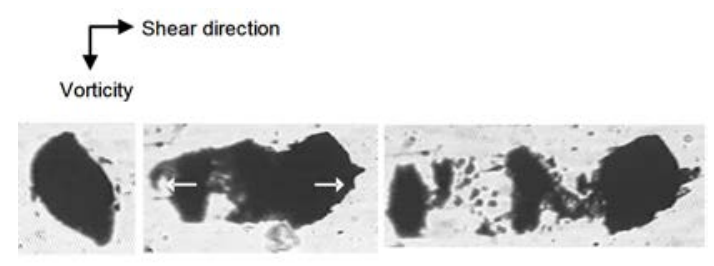

Figure 4

Collin et al., Journal of Applied Polymer Science 

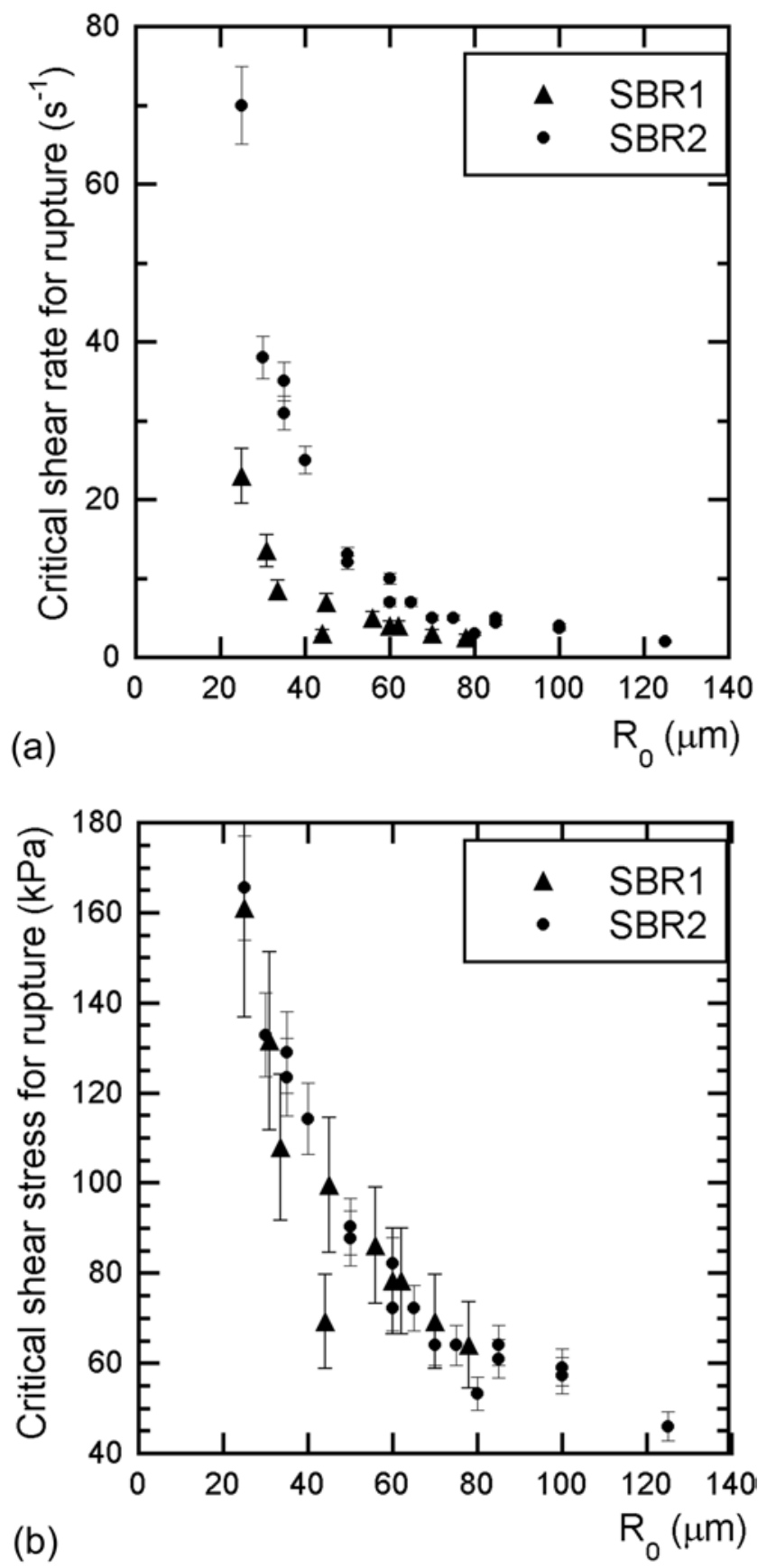

Figure 5

Collin et al., Journal of Applied Polymer Science 


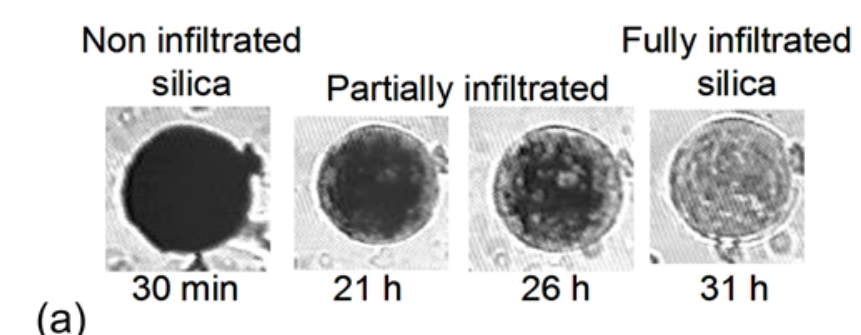

(a)

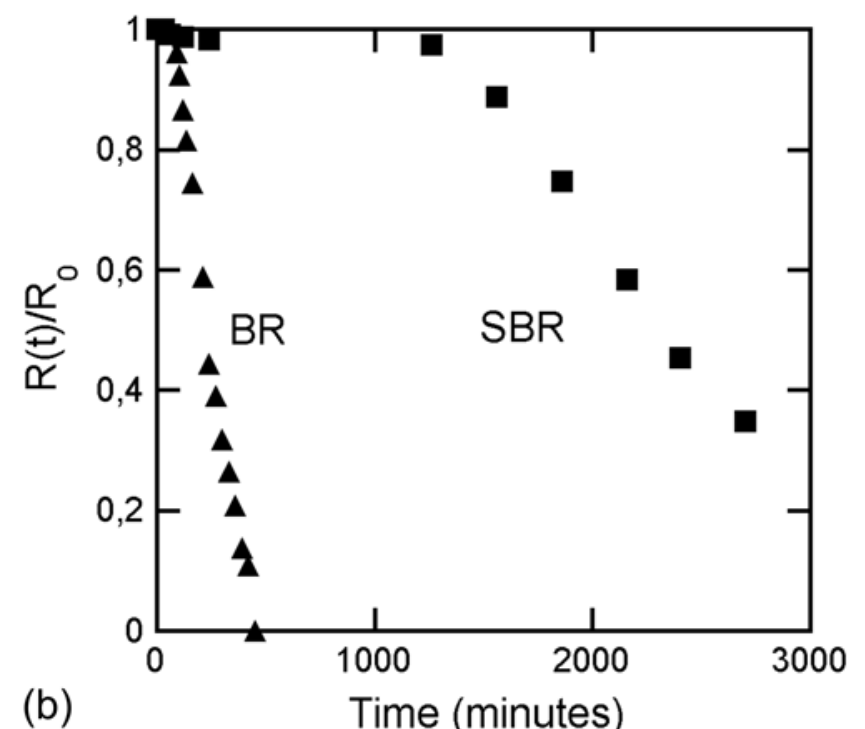

Figure 6

Collin et al., Journal of Applied Polymer Science 


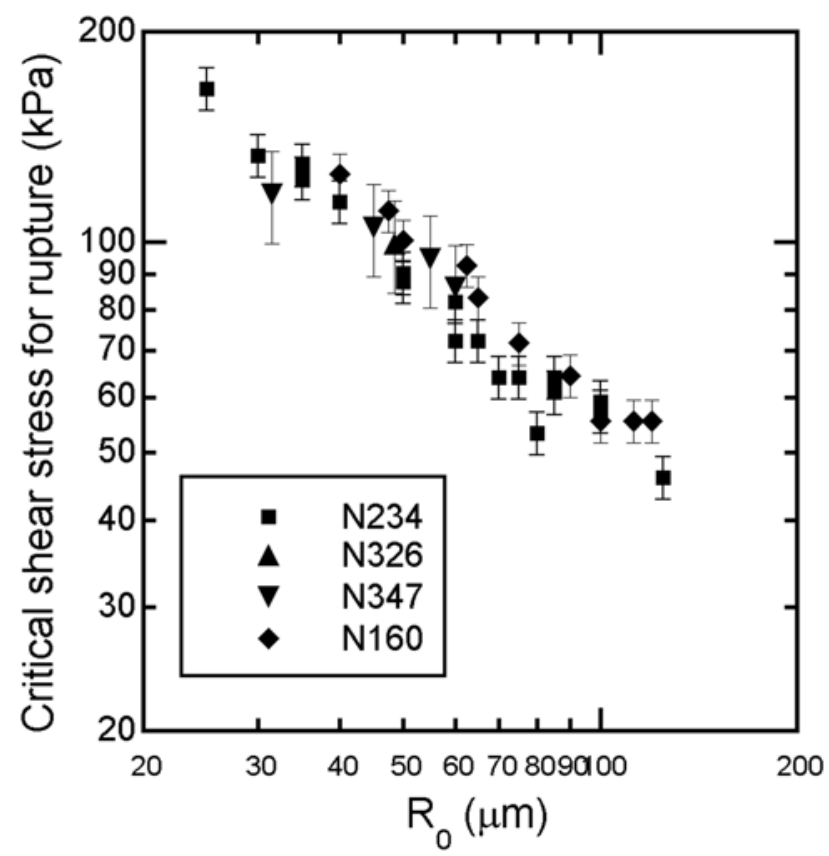

Figure 7

Collin et al., Journal of Applied Polymer Science 


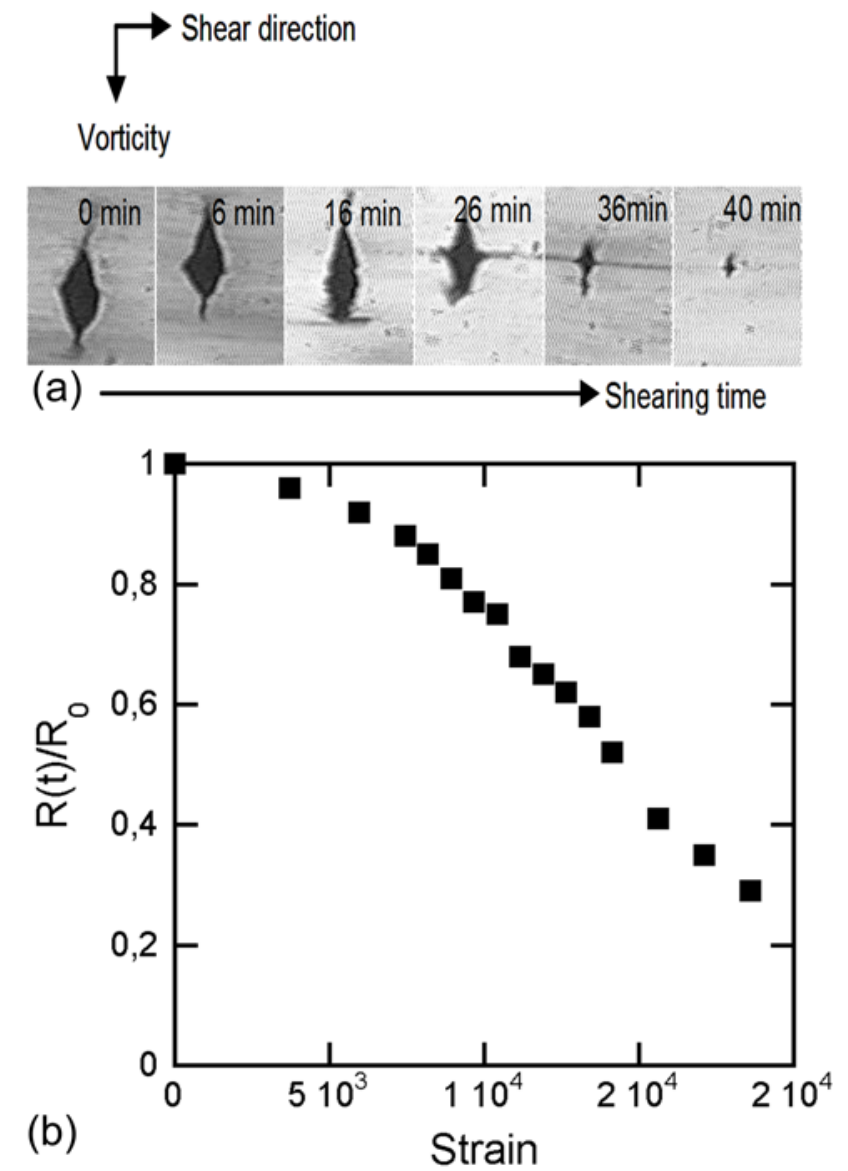

Figure 8

Collin et al., Journal of Applied Polymer Science 


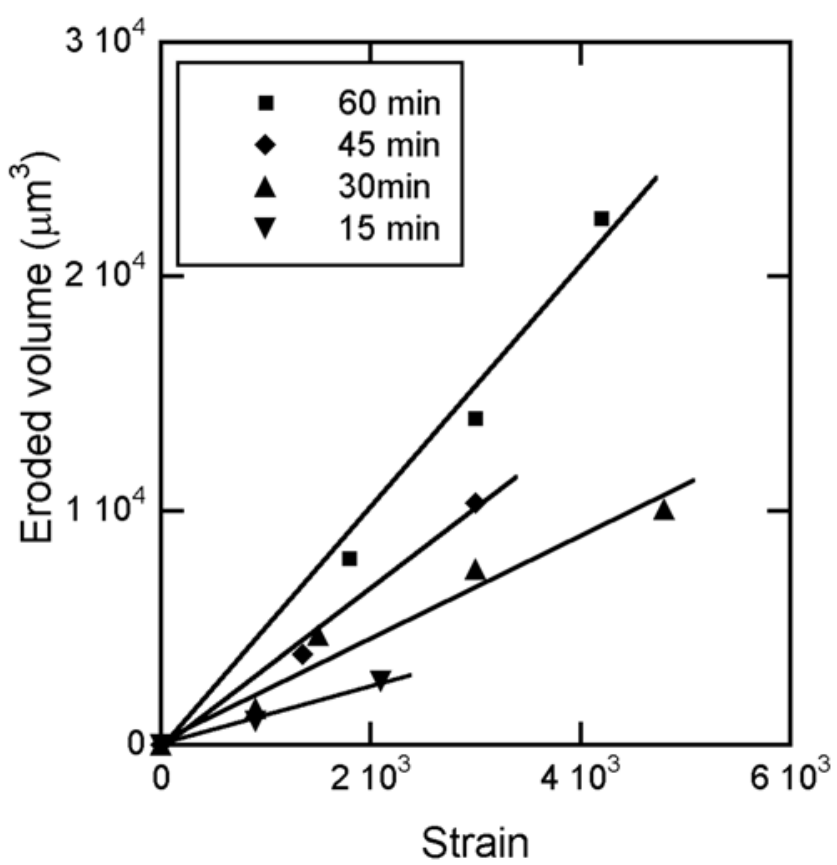

Figure 9

Collin et al., Journal of Applied Polymer Science 

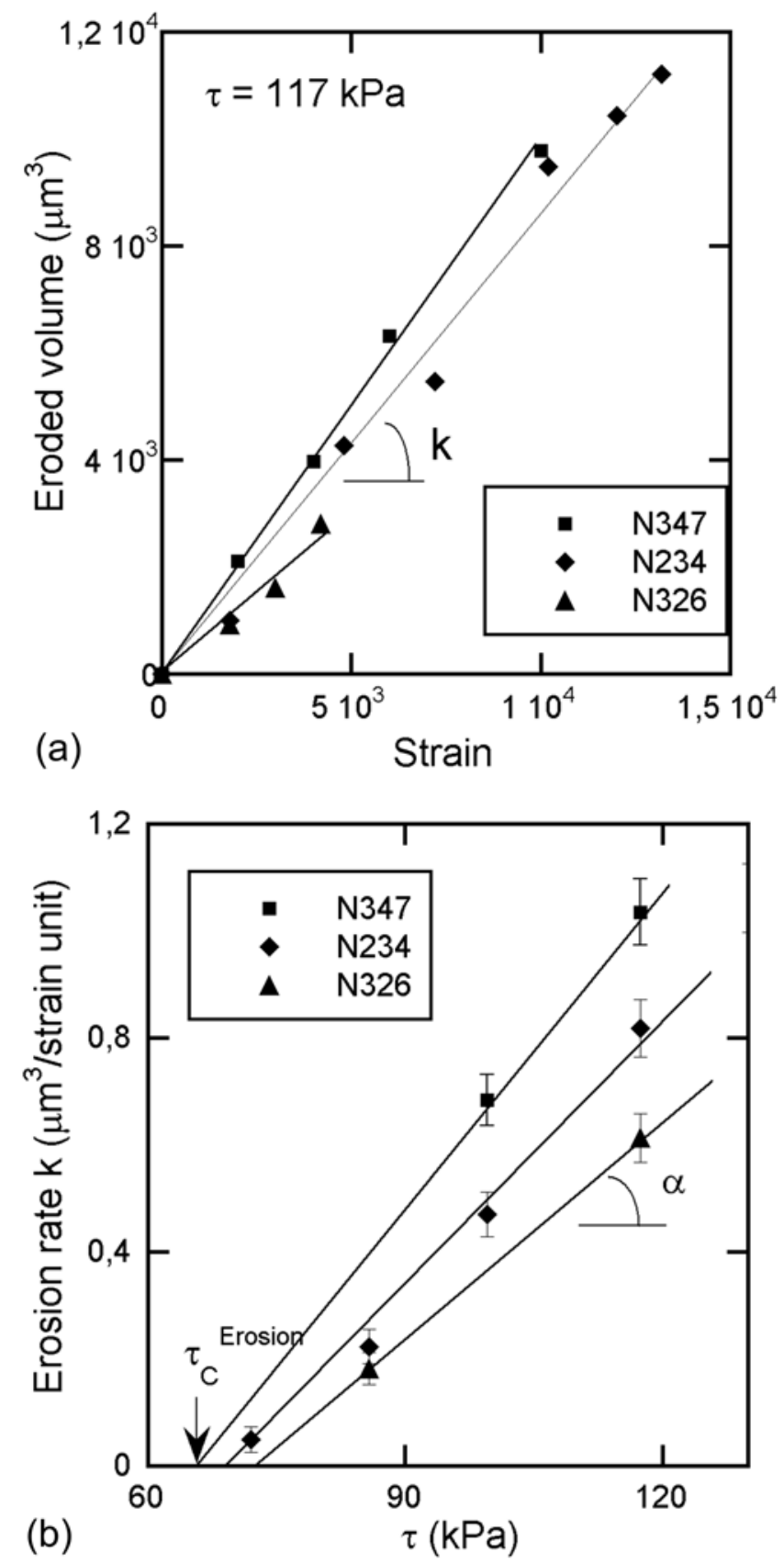

Figure 10

Collin et al., Journal of Applied Polymer Science 


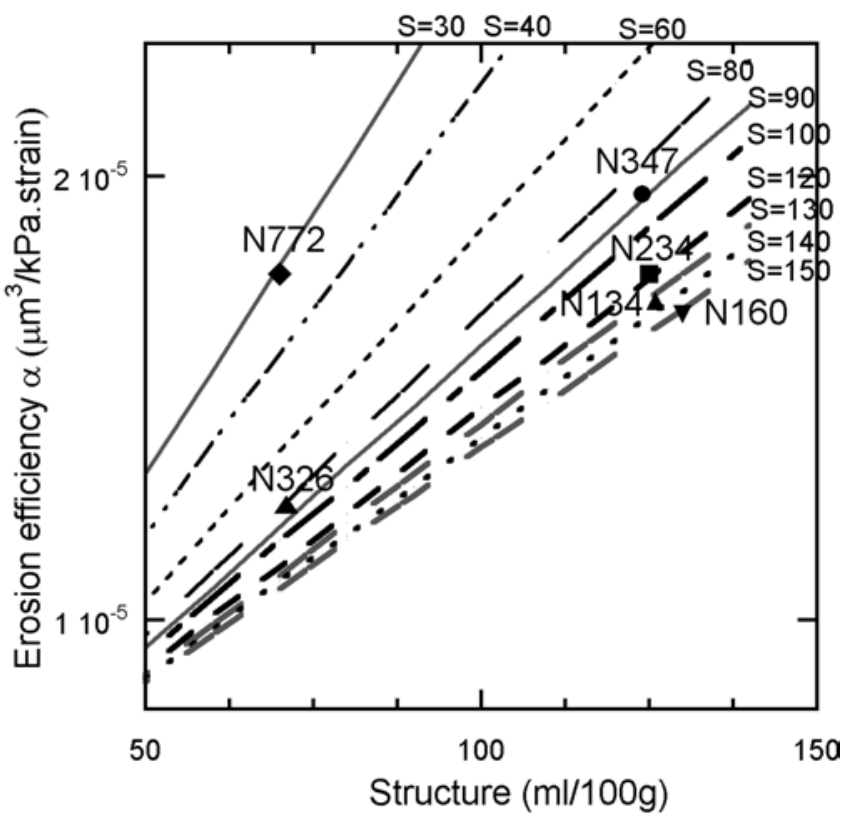

Figure 11

Collin et al., Journal of Applied Polymer Science 